\title{
Relación entre dermatitis atópica y alergia alimentaria
}

\author{
ROSARIO ROJAS A. ${ }^{1}$, ARNOLDO QUEZADA L. ${ }^{2}$ \\ 1. Inmunóloga Hospital Clínico Universidad de Chile. \\ 2. Inmunólogo, Pediatra Neumólogo Departamento Pediatría Sur Facultad de Medicina Universidad de Chile, \\ Hospital Exequiel González Cortés.
}

\begin{abstract}
Relationship between atopic dermatitis and food allergy

The term "allergic march" refers to the history of different atopic manifestations throughout the patient's life. Children with food allergy (FA) are more predisposed to the development of other allergic diseases such as atopic dermatitis $(\mathrm{AD})$, asthma and allergic rhinitis. $\mathrm{AD}$ and FA coexist to a greater extent in patients with early signs of $\mathrm{AD}$, aggressive and persistent symptoms. Meanwhile, FA is a precipitating factor to AD especially in patients with IgE-mediated FA. Correlation to delayed manifestations of FA may also be found. Epithelial barrier dysfunction, mainly attributed to mutations in the filaggrin gene, has been described as a possible trigger for allergen sensitization by increasing skin permeability. This study describes general characteristics of DA and current research evidence regarding the role of FA in the DA development, management and prevention strategies. Also, the utility of diagnostic tests, treatment and prevention in children with DA and FA are discussed. The restoration of impaired skin barrier to prevent sensitization to antigens may have an important role to prevent the development of allergic diseases, especially respiratory diseases.

(Key words: Food allergy, atopic dermatitis, skin tests, specific IgE, patch test, oral challenge, elimination diet).

Rev Chil Pediatr 2013; 84 (4): 438-450
\end{abstract}

\section{RESUMEN}

La denominada "marcha alérgica" se caracteriza por diferentes manifestaciones atópicas relacionadas y sucesivas a lo largo de la vida del individuo. Los niños que presentan alergia alimentaria (AA) tienen mayor predisposición al desarrollo de otras enfermedades alérgicas entre las cuales destacan dermatitis atópica (DA), asma y rinitis alérgica. La DA y AA coexisten en mayor medida en los pacientes que presentan DA de comienzo precoz, agresiva y persistente. Por su parte, la AA es un factor precipitante de DA en un subgrupo de pacientes especialmente aquellos con AA mediadas por IgE, y también existiría correlación con las manifestaciones de AA de tipo retardadas. La disfunción en la barrera epitelial principalmente atribuida a mutaciones en el gen

Recibido el 22 de septiembre de 2012, devuelto para corregir el 09 de noviembre de 2012, segunda versión 24 de octubre de 2012, aceptado para publicación el 16 de diciembre de 2012.

Este trabajo cumple con los requisitos sobre consentimiento /asentimiento informado, comité de ética, financiamiento, estudios animales y sobre la ausencia de conflictos de intereses según corresponda.

Correspondencia a:

Arnoldo Quezada L.

E-mail: aquezada@med.uchile.cl 
de la filagrina se ha descrito como posible desencadenante de la sensibilización a alérgenos por aumento de la permeabilidad cutánea. Se describen las características generales de la DA y evidencias de investigaciones actuales con respecto al rol de la AA sobre el desarrollo de la DA, su manejo y estrategias de prevención. Se discute la utilidad de los exámenes para el diagnóstico y las indicaciones de tratamiento y prevención en el manejo de niños con DA y AA. La restauración de las alteraciones de la barrera cutánea para prevenir la sensibilización antigénica tendría un rol importante para evitar el desarrollo de enfermedades alérgicas especialmente respiratorias.

(Palabras clave: Alergia alimentaria, dermatitis atópica, pruebas cutáneas, IgE específica, test de parche, provocación oral, dieta de eliminación).

Rev Chil Pediatr 2013; 84 (4): 438-450

\section{Introducción}

Tanto la dermatitis atópica (DA) como la alergia alimentaria (AA) son condiciones que con frecuencia señalan el comienzo de la denominada "marcha alérgica", que se caracteriza por diferentes manifestaciones atópicas relacionadas y sucesivas a lo largo de la vida del individuo. Comúnmente DA y AA coexisten en mayor medida en los pacientes que presentan DA de comienzo precoz, agresiva y persistente $^{1-3}$.

La asociación de DA y AA se estima en alrededor del 35\% de los niños que presentan DA. En la mayoría de los pacientes ambas condiciones son transitorias y mejoran de manera espontánea en la adolescencia. Los pacientes adultos que persisten con DA sólo en un escaso porcentaje presentarán simultáneamente $\mathrm{AA}^{2}$.

La AA es un factor desencadenante de exacerbaciones de DA en un subgrupo de pacientes con DA especialmente aquellos con AA mediada por IgE, sin embargo, también existiría cierta correlación con las manifestaciones de AA de tipo retardadas ${ }^{2}$.

Existe evidencia emergente sobre la existencia de una disfunción en la barrera epitelial principalmente dada por mutaciones en el gen de la filagrina (FLG), una proteína estructural epidérmica que forma parte del factor humectante natural, condición que determina una función anormal a nivel de la piel. Esta mutación se ha descrito como posible desencadenante de la sensibilización a alergenos debida al aumento de la permeabilidad cutánea lo que explicaría la presencia simultánea de DA y AA.
Muchos de los niños que desarrollan DA tienen mayor predisposición al desarrollo de otras enfermedades alérgicas entre las cuales destacan alergia alimentaria, asma y rinitis alérgica. Alrededor de un 50\% de los pacientes con DA desarrollarán otros tipos de manifestaciones atópicas ${ }^{1,4,5}$ (figura 1).

El propósito de este artículo es revisar las características generales de la DA y evidencias de investigaciones actuales con respecto a la relación entre las manifestaciones de DA y AA, su manejo y estrategias de prevención. La información se obtuvo mediante búsqueda en las bases de datos MEDLINE/PubMed y Cochrane Library y se revisaron publicaciones recientes accesibles en idioma inglés, en texto completo.

\section{Dermatitis atópica (DA)}

La DA es una enfermedad inflamatoria y pruriginosa de la piel, de curso crónico y recidivante, desencadenada por diversos factores precipitantes que actúan sobre un terreno congénitamente alterado. Los pacientes con DA refieren con frecuencia antecedentes personales o familiares de asma, rinitis alérgica o dermatitis atópica, y presentan niveles séricos de IgE elevados ${ }^{1}$.

La prevalencia a nivel mundial de DA se calcula entre un 10 a $30 \%$ en niños y entre 1 y $3 \%$ en adultos. Estas variaciones dependen del grupo examinado, país, clima y método de recolección de la información, entre otros ${ }^{6,7}$.

La gran mayoría de los pacientes presentan la forma leve o moderada, siendo muy bajo el 


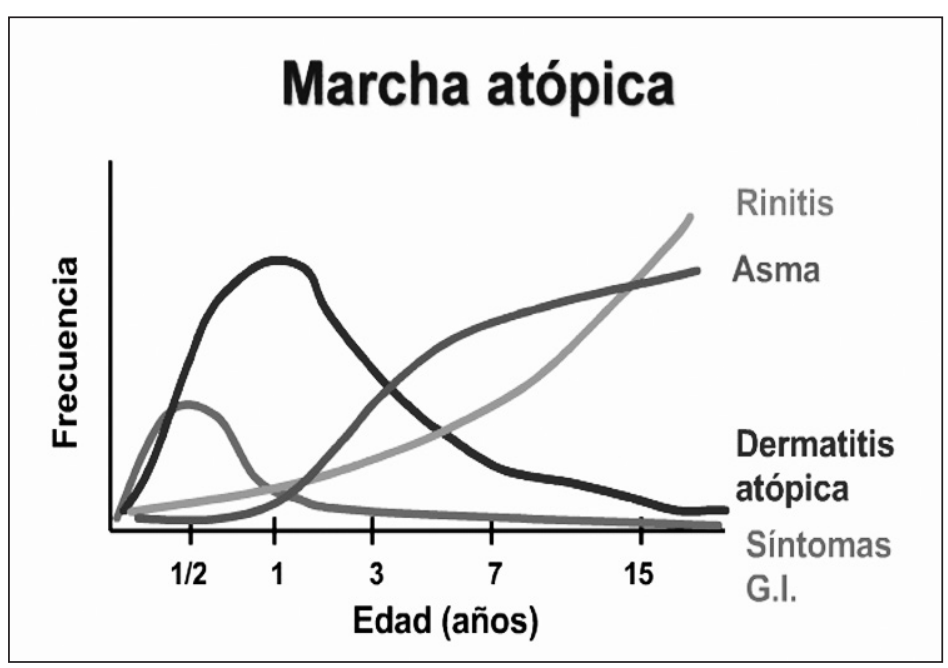

Figura 1. Marcha Atópica, historia natural de las enfermedades alérgicas. (Modificado de The allergy march, Sampson, 2002). porcentaje de casos graves. El 65\% de los casos comienzan en el primer año de vida. En el $85 \%$ de los pacientes las lesiones se desarrollan antes de los 5 años de edad. Entre el 50 a $70 \%$ de los pacientes que inician la enfermedad en la infancia, presenta remisión durante la adolescencia. Sólo el 2\% de los casos nuevos ocurren después de los 20 años ${ }^{8,9}$.

Las cifras de prevalencia se han incrementado 2 a 3 veces durante las tres últimas décadas en países industrializados. La amplia variación en la prevalencia dada principalmente entre las zonas urbanas y rurales identificadas dentro de la población de un mismo país, incluso en grupos étnicos similares, sugiere que los factores ambientales determinan en gran medida la expresión de la $\mathrm{DA}^{1,2,9,10}$.

La condición socioeconómica también tendría un rol sobre su prevalencia, pues un nivel socioeconómico alto se correlaciona con un incremento en la prevalencia de DA. Por otra parte, el tamaño familiar influye, ya que grupos familiares numerosos se asocian a una disminución de la prevalencia de DA. En conjunto, este tipo de comportamiento puede explicarse por la aparición de factores de riesgo asociados a un estilo de vida moderna y la "hipótesis de la higiene" ${ }^{\text {1,2,9-11. }}$.

\section{Clínica de la $\mathrm{DA}$}

La DA ofrece un amplio rango de manifestaciones clínicas. Entre las más comunes se incluyen lesiones eccematosas recidivantes o crónicas, muy pruriginosas, con una distribución típica según la edad, constituyendo elementos esenciales para el diagnóstico.

La distribución de las lesiones varía según la edad de los pacientes. Se distinguen tres etapas, las que pueden superponerse y presentar períodos de remisión variable. Durante la etapa del lactante y primera infancia (desde los 2 a 6 meses hasta los 2 años) la DA es generalmente más aguda y afecta principalmente la cara, cuero cabelludo y superficies extensoras de las extremidades. En la etapa infantil (entre los 2 y 10 años de edad) los niños que han estado afectados por largos períodos de tiempo presentan liquenificación de las lesiones y localización a nivel de los pliegues flexores de las extremidades. En la etapa del adolescente (entre los 10 y 20 años) y adulto joven la DA tiende a comprometer las superficies flexoras, además de las manos y los pies ${ }^{8,9}$.

El prurito crónico lleva al rascado que es responsable de la producción inicialmente de lesiones primarias como eritema, pápulas y vesículas y luego de cambios secundarios como erosiones, alteraciones pigmentarias y aumento del grosor y cuadriculado normal de la piel (liquenificación) ${ }^{9}$.

La sequedad de la piel o xerosis es una manifestación característica de esta enfermedad. Además, existen ciertos estigmas que aunque inespecíficos suelen ser frecuentes en estos pa- 
cientes como la hiperlinearidad palmo-plantar, pliegue infraorbitario, dermografismo blanco, palidez facial, hiperpigmentación periorbitaria, disminución o ausencia del borde lateral de las cejas, línea de implantación baja del pelo e hiperqueratosis folicular ${ }^{10}$.

Los criterios clínicos más usados en el diagnóstico de DA corresponden a los criterios de Hanifin y Rajka (tabla 1). Otros criterios más simplificados fueron publicados por un grupo de expertos europeos (tabla 2).

\section{Histopatología de la $\mathrm{DA}$}

Las lesiones eccematosas agudas se caracterizan por un marcado edema intercelular epidérmico llamado espongiosis, con un infiltrado de células presentadoras de antígeno (CPA) y moléculas de IgE. A nivel de la dermis se puede observar infiltrado de linfocitos T (LT) CD4+ activados con fenotipo de memoria. Las lesiones crónicas de DA presentan remodelación tisular dada por inflamación crónica. Las lesiones liquenificadas presentan una epidermis acantótica (separación de células epidérmicas) con elongación de surcos y mínima espongiosis. A nivel de la dermis se encuentra infiltrado mononuclear de predominio macrofágico. Las lesiones crónicas contienen eosinófilos, células que contribuyen a la inflamación y a la injuria del tejido, a través de la producción de radicales de oxígeno, citoquinas proinflamatorias y liberación de proteínas tóxicas de los gránulos. La piel de pacientes con DA sin lesiones aparentes también presenta alteraciones histológicas ${ }^{12}$.

\section{Inmunopatogenia de la $\mathrm{DA}$}

La DA es considerada una enfermedad multifactorial ya que no se ha podido establecer un concepto etiopatogénico único. La DA sería el resultado de una compleja interacción entre susceptibilidad genética, medio ambiente, defectos en la barrera de la piel y alteraciones en la respuesta inmune local y sistémica. La DA ha sido propuesta como una manifestación cutánea de un desorden sistémico que también incluye otras entidades como asma, alergia alimentaria y rinitis alérgica. Estas condiciones se caracterizan, por un incremento en los nive-
Tabla 1. Criterios diagnósticos de dermatitis atópica según Hanifin y Rajka

Han de cumplirse 3 o más criterios mayores y 3 o más criterios menores

\section{Criterios mayores}

Prurito

Morfología y distribución característica: Liquenificación en flexuras en adultos, compromiso de cara, pliegues y superficies extensoras en jóvenes y niños, combinación de estos patrones en niños y jóvenes

Carácter crónico y recidivante

Historia personal o familiar de atopia

\section{Criterios menores}

Xerosis, Ictiosis/exageración pliegues palmares, queratosis pilar, Reactividad cutánea inmediata, Elevación valores séricos de IgE, Edad precoz de comienzo, Tendencia a infecciones cutáneas, Eczema de pezón, Queilitis, Conjuntivitis recidivante, Pliegue infraorbitario de Dennie Morgan, Ojeras u oscurecimiento periocular, Palidez facial o eritema en cara, Pitiriasis alba, Pliegues en parte anterior del cuello, Prurito provocado por la sudoración, Intolerancia a la lana y solventes, Acentuación perifolicular, Intolerancia a algunos alimentos, Evolución influenciada por factores ambientales y emocionales, Dermografismo blanco

Fuente: Hanifin J, Rajka G. Diagnosis features of atopic dermatitis. Acta Derm Venereol (Suppl) 1980; 92: 44-47.

Tabla 2. Criterios diagnósticos establecidos por el grupo del Reino Unido

Historia de prurito asociado a 3 o más de los siguientes:

a. Antecedentes de piel seca generalizada

b. Dermatitis flexural visible

c. Comienzo antes de los 2 años de edad

d. Antecedentes personales de asma o rinitis (o familiares de primer grado en menores de 4 años)

Fuente: Brenninkmeijer $\mathrm{E}$, Schram M, Leeflang $\mathrm{M}$, et al. Diagnostic criteria for atopic dermatitis: a systematic review. Br J Dermatol 2008; 158: 754

les séricos de IgE total y eosinofilia periférica. Adicionalmente, numerosos estudios epidemiológicos han establecido una fuerte asociación entre DA, asma y rinitis alérgica. Entre el $20 \%$ y el $60 \%$ de los casos de DA se asocia a manifestaciones respiratorias (asma-rinitis) y el $40-67 \%$ de los pacientes presentan antecedentes personales y/o familiares de atopia ${ }^{13-15}$.

Aproximadamente un $80 \%$ de los pacientes con DA presentan un aumento de los niveles séricos de IgE. Sin embargo, debido a que este marcador no está presente en todos los casos de DA, esta enfermedad ha sido dividida en 2 subtipos $^{11-13,16-18}$. 
a) DA extrínseca (alérgica): afecta entre el $70 \%$ y $80 \%$ de los pacientes. Existe una sensibilización a alérgenos ambientales por lo que cursa con aumento de los niveles de $\mathrm{IgE}$ sérica.

b) DA intrínseca o no alérgica: afecta entre el $20 \%$ a $30 \%$ de los pacientes, no existe sensibilización a alérgenos ambientales, y cursan con niveles normales de IgE.

A pesar de esta división, los 2 subtipos de DA tienen presentaciones clínicas indistinguibles en términos de tipo y distribución de lesiones, infiltración de LTCD4+ y otras características histológicas. Consecuente con esto, se plantea la hipótesis que la sensibilización por IgE no es un requisito necesario para el desa- rrollo de lesiones eccematosas de la piel con características de $\mathrm{DA}^{12,16}$. Según este planteamiento, otras alteraciones inmunológicas descritas en los pacientes con DA intrínseca serían suficientes para producir el desarrollo de esta patología. Por otra parte, pacientes con DA extrínseca presentan fenotipos clínicos más severos que los pacientes con DA intrínseca, sugiriendo que la sensibilización por IgE es un índice de gravedad en esta enfermedad ${ }^{1,4}$.

La DA se caracteriza por ser una enfermedad bifásica (figura 2), con una etapa inicial en la que predomina la respuesta LT helper tipo 2 (Th2) y una segunda fase crónica, eczematosa, donde predomina una respuesta $T h 1^{13}$.

La disfunción de la barrera epitelial causada por mutaciones del gen de la filagrina (FLG),

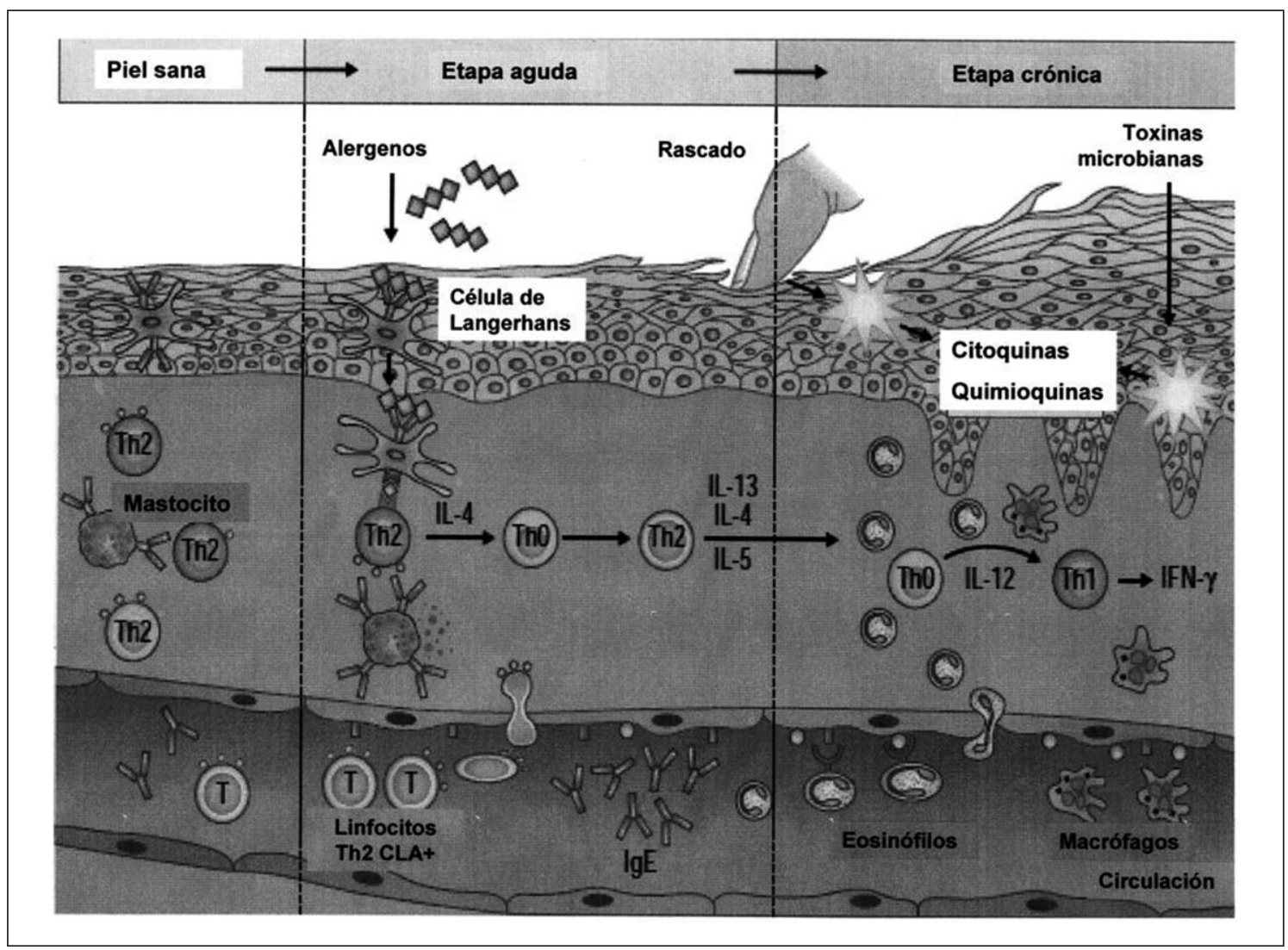

Figura 2. Patogenia de la dermatitis atópica: progresión según tiempo evolución. En el esquema se observan las principales citoquinas y subtipos celulares involucrados y el cambio del perfil atópico a uno de tipo inflamatorio con el transcurso de la enfermedad. En la primera etapa de la enfermedad el tipo celular preponderante corresponde a los linfocitos T helper tipo 2 y la secreción de citoquinas IL-4, 5 y 13 lo que lleva a una producción preferencial de IgE; posteriormente en la etapa de cronicidad de la enfermedad existe un infiltrado celular de tipo inflamatorio con linfocitos T helper tipo 1 y acumulación de macrófagos y citoquinas proinflamatorias como IL-12 e IFN gamma (Modificado de Leung DYM. J Allergy Clin Immunol 2000). 
una proteína estructural epidérmica que forma parte del factor humectante natural, se considera un factor de riesgo emergente para la DA severa de comienzo precoz ${ }^{1,6}$. El gen de la FLG reside en el cromosoma 1q21 dentro del complejo de diferenciación epidermal. Las mutaciones del gen de la FLG se han detectado en alrededor de $12 \%$ a $15 \%$ de la población general. Estudios genético-epidemiológicos muestran una importante asociación entre los defectos del gen de la FLG con el aumento del riesgo de padecer DA (Odds ratio 1,99, IC 95\% 1,72-2,31). Además se encontró asociación entre los defectos en el gen de la FLG y una presentación precoz, persistente y más severa de $\mathrm{DA}^{2}$.

\section{Alergia alimentaria (AA)}

AA se define como una reacción adversa que presenta un individuo tras la ingesta de un alimento, producida por reacciones de hipersensibilidad mediadas por IgE, mediadas por células o mixtas (tabla 3 ). Su prevalencia general en EE.UU. es de alrededor del $4 \%$ y varia dentro de los grupos etarios, siendo en niños de $6-8 \%$ y en adultos de alrededor de un 3\%. Un $90 \%$ de las AA se concentran en los alergenos: huevo, leche de vaca, soya, pescado, trigo, maní y nueces.

La AA involucra la combinación de una sensibilización antígeno específica, la presencia de una reacción de tipo IgE, mixta o celular además de una reacción clínica atribuible a ese alergeno particular. La sensibilización por sí sola no significa alergia alimentaria, situación que puede ser clínicamente irrelevante e implica que el diagnóstico de AA no se puede realizar frente a la positividad de un prick test, IgE especifica y/o test de parche positivo ${ }^{1}$.

\section{Rol de la $A A$ en $D A$}

El primer reporte de DA provocada por AA fue descrito por Schloss en 1915, como una erupción cutánea eczematosa en respuesta a alimentos, que mejoraba frente a la eliminación de éstos. A la fecha múltiples estudios de reportes de casos, pruebas controladas con placebo y provocación oral entre otros han demostrado la asociación entre DA y AA.

En enfermos con AA se ha reportado aso-
Tabla 3. Mecanismos inmunológicos de alergia alimentaria

Inmunopatología/enfermedad
Mediado por IgE
Urticaria/angioedema
Reacción gastrointestinal inmediata
Síndrome de alergia oral
Rinitis
Asma
Anafilaxia
Anafilaxia por ejercicio
Asociado a IgE y celular/mixtos
Dermatitis atópica
Gastroenteropatías eosinofílicas
No asociado a IgE
Enterocolitis a proteínas alimentarias
Proctitis a proteínas alimentarias
Enteropatía por proteínas alimentarias
Enfermedad celíaca
Dermatitis herpetiforme
Dermatitis de contacto
Hemosiderosis pulmonar

ciación con DA en cifras tan variables como $35 \%$ a $71 \%{ }^{19,20}$. Cuando se asocian DA y AA, hasta un $75 \%$ de los sujetos puede tener otra condición atópica ${ }^{21}$. Se ha descrito prevalencia entre $30 \%$ y $40 \%$ de AA en enfermos con DA moderada a severa y en estos enfermos la AA mediada por IgE tiene significancia clínica cuando existe historia evidente de reacción inmediata a alimentos, o son evaluados por correlación con síntomas, pruebas cutáneas, IgE específica o pruebas de provocación oral ${ }^{22}$.

Las manifestaciones cutáneas son una de las formas de presentación más frecuente de la AA e incluye a la DA como consecuencia de una reacción mixta en que participan mecanismos mediados por IgE y celulares. Las mutaciones de la FLG en la piel puede aumentar el riesgo de sensibilización alergénica transcutánea y el desarrollo de AA en enfermos con $\mathrm{DA}^{23}$.

Según el informe del panel de expertos convocados por el National Institute of Allergy and Infectious Diseases de Estados Unidos, DA y AA tienen una alta asociación ${ }^{7}$, y aunque el rol de la AA en la patogenia y en la severidad de la DA sigue siendo controvertido, en algunos enfermos pediátricos sensibilizados los alergenos alimentarios son capaces de inducir lesiones urticariales, prurito y exacerbación del eczema, elementos que pueden agravar 
Tabla 4. Asociación entre dermatitis atópica y alergia alimentaria

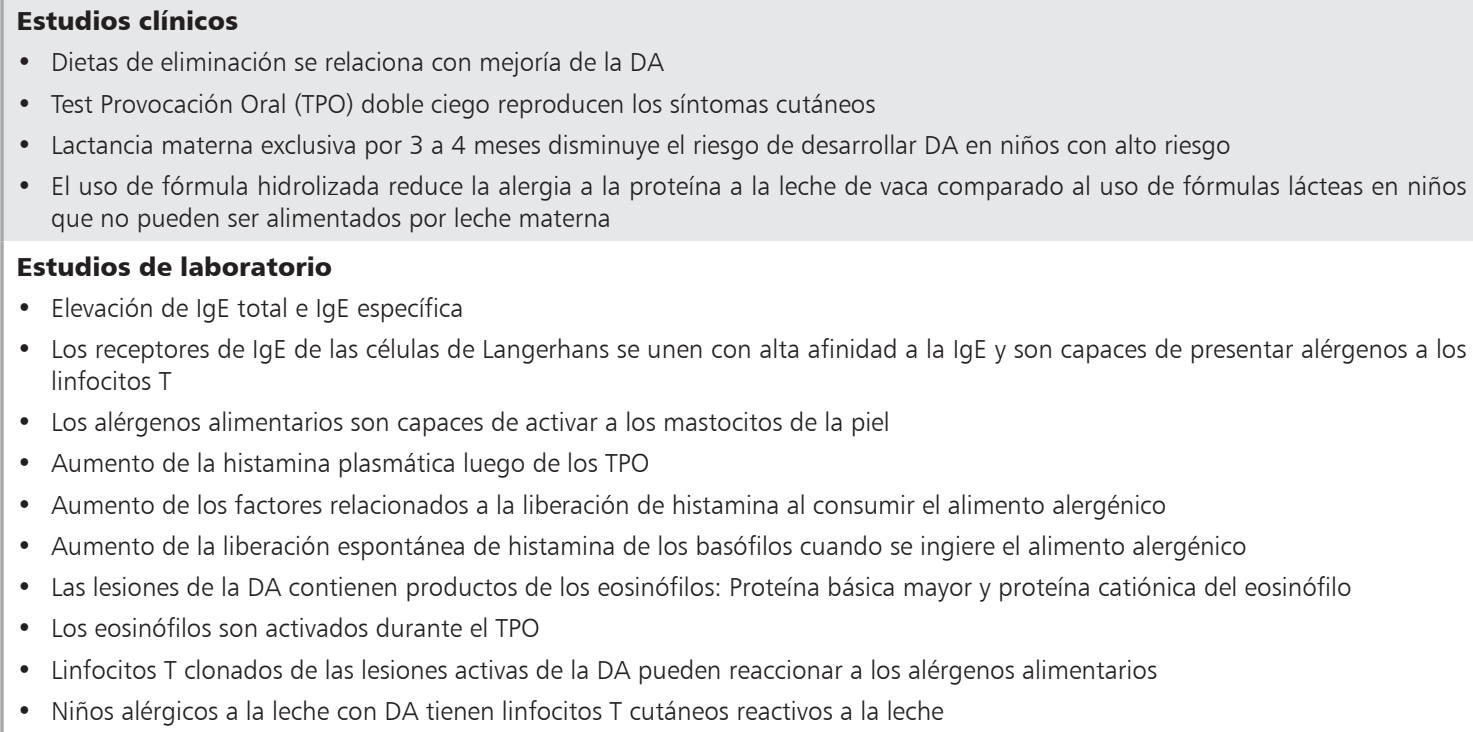

la $\mathrm{DA}^{24}$. La asociación entre DA y diferentes formas clínicas de AA por hipersensibilidad mixtas y no mediadas por IgE como esofagitis eosinofílica, proctocolitis o enterocolitis inducida por alimentos se ha descrito con porcentajes muy variables que van entre $4 \%$ y $65 \% 25-28$.

Los estudios de prevalencia de AA confirmada con provocación doble ciego placebo controlada muestran que un $35 \%$ de los pacientes con DA sensibilizados a alimentos tendrían una AA relevante frente a la prueba de provocación oral ${ }^{1}$.

Múltiples estudios han demostrado la conexión entre la ingesta alimentaria, desarrollo de síntomas y el aumento de la liberación de mediadores presentes en la DA (tabla 4). Como ejemplo, Sampson demostró que pacientes con DA e IgE especifica positiva para algún alergeno alimentario, tras someterse a una prueba de provocación oral muestran aumento de las concentraciones séricas de histamina en comparación a controles. Posteriormente demostró que la ingesta crónica del alergeno alimentario se asocia a una mayor liberación de histamina de manera espontánea por basófilos en cultivos celulares de pacientes con DA versus controles. Otros estudios han demostrado el aumento de mediadores de eosinófilos frente a la exposición a alergenos alimentarios ${ }^{1,29,30}$.
Se ha descrito la presencia de linfocitos $\mathrm{T}$ específicos para diversos antígenos alimentarios (maní, ovoalbúmina, caseína) en el suero de pacientes con DA inducida por alimentos. Linfocitos T cutáneos específicos para antígeno han sido clonados desde biopsias de piel en pacientes con DA tanto en piel sana como en piel con lesiones. Se han identificado LT antígeno específicos con marcadores de superficie denominados CLA (cutaneous leukocyte antigen) que son capaces de interactuar con E-selectina, una molécula de adhesión celular, para dirigir el tráfico selectivo ("homing") de los LT a la piel lo que explica como los antígenos alimentarios tendrían su efecto en la llegada de los LT a su órgano diana ${ }^{1}$.

\section{Evaluación del paciente con DA y sospecha de AA}

Las guías clínicas del NIAID recomiendan que el diagnóstico de AA debe ser realizado frente a la combinación de historia clínica, pruebas cutáneas y/o IgE específica asociado a una confirmación mediante la prueba de provocación oral positiva para el alérgeno en estudio ${ }^{7}$. No se recomienda la prueba de provocación oral en sujetos con antecedentes de reacciones alérgicas severas o cuando la his- 
toria clínica es claramente concordante con el antígeno sospechado ${ }^{1}$. Alrededor del $80 \%$ de los pacientes con DA tendrán IgE especifica positiva para alergenos alimentarios o pólenes, en muchas ocasiones multisensibilizados siendo pocas de estas sensibilizaciones clínicamente relevantes. Dado lo anterior deben tomarse precauciones antes de considerar un test positivo relevante en el contexto de DA. Los test cutáneos negativos tienen un valor predictivo negativo (VPN) que alcanza el 95\% para una alergia, sin embargo, el valor predictivo positivo (VPP) no supera el $60 \%$. Test serológicos de IgE especifica comparten similares VPN y VPP con los test cutáneos.

Los alérgenos alimentarios más relevantes en DA son huevo, leche de vaca, trigo, soya y maní siendo responsables del $75 \%$ de la DA asociada a $\mathrm{AA}^{1,7}$. El diagnóstico certero es sin embargo, engorroso dado que se deben excluir los factores confundentes que llevan a mala interpretación subjetiva. La latencia en la manifestación de los síntomas en la DA asociada a AA que puede incluso llegar a las $72 \mathrm{~h}$ post prueba de provocación dificulta la observación; sólo un $25 \%$ se presenta dentro de las primeras $2 \mathrm{~h}$ desde la ingesta. Los resultados de una exposición también varían con respecto a la exposición crónica al alimento.

La historia familiar de atopia y la presencia de DA son factores de riesgo para desarrollar tanto sensibilización a alimentos como AA sintomática, según datos obtenidos en al menos 3 estudios. En uno se encontró que 25\% a 33\% de niños menores de 5 años con DA moderada a severa tenían AA mediada por IgE confirmada por la presencia de IgE específica, pruebas de provocación oral o historia consistente con reacción alérgica al alimento ${ }^{22}$. En otro estudio, $82 \%$ de 138 enfermos con alergia al maní tenían $\mathrm{DA}^{31}$. Finalmente en el tercer estudio, los enfermos que presentaban DA severa en los primeros 3 meses de vida tenían con mayor frecuencia $\mathrm{IgE}$ específica a leche de vaca, huevo y maní sugiriendo que este grupo tiene mayor riesgo de manifestar AA mediada por $\mathrm{IgE}^{32}$. Estos estudios sugieren fuertemente que AA y DA moderada a severa se presentan con frecuencia en un mismo niño y que la DA severa de comienzo precoz se asocia con riesgo alto de sensibilización a alimentos. Otro estudio sugiere que la sensibilización a maní se asocia independientemente con dermatitis periarticular y en pliegues cutáneos, rasgos que caracterizan a la $\mathrm{DA}^{33}$. El mecanismo de sensibilización temprana a alimentos no está claro.

La gravedad de la reacción alérgica a alimentos es multifactorial y variable, pero la presencia de DA puede condicionar una absorción de alergenos más rápida y ser otro factor que favorece una mayor severidad ${ }^{34,35}$.

Si la DA persiste a pesar de manejo óptimo y tratamientos tópicos, o si existe historia evidente de reacción inmediata tras la ingesta de un alimento específico, los expertos sugieren realizar pruebas de AA para leche, huevo, maní, trigo y soya en niños menores de 5 años con DA moderada a severa. El diagnóstico precoz puede facilitar un mejor manejo y reduce el riesgo de exposición a alergenos alimentarios, recordando que dietas muy restrictivas pueden tener riesgos y que para eliminar un alimento totalmente de la dieta debe haber seguridad que el niño es alérgico a ese alimento. Cuando existe historia de una probable reacción retardada a alimentos se requiere una mayor evaluación clínica por especialista y otras pruebas diagnósticas para AA.

Se requiere una historia clínica acuciosa, tratando de consignar el alimento sospechoso de las manifestaciones clínicas, temporalidad y su recurrencia frente a reexposición. Se recomienda realizar test cutáneo y/o serológico sólo a los alergenos identificados como relevantes en la historia clínica dado su alto número de falsos positivos y bajo valor predictivo positivo. El test cutáneo permite la exclusión de hipersensibilidad inmediata en la mayoría de los casos que es negativo. La IgE específica sérica presenta valores variables entre estudios, pudiendo tener según algunos resultados mejor VPP que el test cutáneo. Su utilidad radica en los menores de 2 años donde el test cutáneo es menos aplicado y para confirmar reacciones de hipersensibilidad tipo $\mathrm{I}^{1,2}$.

Existen puntos de corte con valores diagnósticos para el 95\% de los pacientes en estudios descritos tanto para el diámetro del eritema en test cutáneo como para el título de IgE sérica, sin embargo, éstos no están estan- 
darizados y no siempre llevan a un diagnóstico certero ya que no son extrapolables a todas las poblaciones. Podrían ser útiles frente a reacciones severas donde la provocación oral lleva a efectos adversos desproporcionados.

El test de parche, aumenta la sensibilidad y especificidad de IgE y prick test especialmente en reacciones tardías mediadas por hipersensibilidad celular; sin embargo, requiere de estandarización y no se ha comprobado su rol definitivo en el diagnóstico de DA asociada a AA. El test de parche es un método útil en la investigación de hipersensibilidad retardada a alimentos en niños pequeños y como ayuda para identificar alergenos específicos para la indicación de dieta de exclusión, sin embargo, la relevancia clínica de esta prueba debe ser confirmada con dieta de exclusión y provocación.

Los test cutáneos pueden permanecer positivos en el tiempo, aun cuando haya desaparecido la DA frente a la ingesta del alimento sospechado, sin embargo, los títulos de $\operatorname{IgE}$ especifica tienden a descender en conjunto con la aparición de tolerancia ${ }^{1,2,6}$.

\section{Test de provocación oral (TPO)}

La recomendación general es realizar un TPO después de una dieta de eliminación de al menos 2 sem; al mismo tiempo con la dieta de eliminación debe iniciarse un tratamiento cutáneo agresivo e intensivo lo que permite las mejores condiciones de la piel para realizar y evidenciar las consecuencias del TPO. Durante el TPO se acepta la mantención de corticoides tópicos de baja potencia sin embargo, hay que suspender la fototerapia y el tratamiento con antihistamínicos. Existen protocolos de TPO publicados $^{36}$. Tras TPO se debe observar la piel considerando un mínimo de evaluación a las 48 $\mathrm{h}$ post ingesta, se recomienda el uso de score estandarizado de la piel (ej. SCORAD). Como TPO ideal se recomienda el método placebo doble ciego controlado, lo cual no siempre es posible llevar a cabo en la práctica clínica dado su alto costo monetario y consumo de tiempo.

\section{Dieta de eliminación}

Como tratamiento más efectivo de la AA se propone la dieta de eliminación, sin embargo, en muchos casos ésta es mal guiada y tiene una restricción incompleta lo que lleva a la frustración dado la escasa respuesta clínica. Parecen no existir beneficios con la utilización de la dieta básica o con dieta con pocos alimentos en personas no seleccionadas con eccema atópico $^{37,38}$.

En el manejo de niños con pruebas cutáneas o serológicas positivas, se debe considerar una prueba con dieta de eliminación, con un intervalo de tiempo limitado para evaluar la repercusión clínica de la restricción. Se deben a su vez reconsiderar los requerimientos nutricionales y programar un esquema suplementario adecuado según los déficits producidos por la dieta de eliminación. Si los resultados son satisfactorios con la dieta de eliminación se debe realizar una provocación oral (TPO) en estado asintomático para descartar efecto placebo y confirmar el diagnóstico ya que el TPO corresponde al "gold standard" diagnóstico de la alergia alimentaria.

En enfermos con AA demostrada que además presentan DA, se recomienda la eliminación de los alergenos específicos, aunque existen datos limitados en este tema. La dieta de eliminación puede disminuir la severidad de la DA, pero no hay evidencia suficiente que demuestre que la evitación de los alergenos alimentarios altere el curso de la enfermedad. En niños sin AA demostrada las dietas de eliminación no están recomendadas en el manejo de la DA u otras condiciones atópicas, incluso tal conducta puede ocasionar deficiencias nutricionales y de crecimiento.

Se han publicado al menos dos revisiones sistemáticas de alta calidad que evalúan el efecto de la dieta de exclusión en el tratamiento de la DA. El primero no encontró beneficios significativos en la prevención de eczema en lactantes cuyas madres cumplieron una dieta de exclusión de alergenos alimentarios durante la lactancia en comparación con madres que recibieron alimentación sin restricciones dietéticas ${ }^{38}$. El otro metanálisis no encontró evidencias para recomendar dietas de exclusión en el tratamiento de DA de enfermos no seleccionados ${ }^{37}$.

La reintroducción de alimentos en la dieta en niños previamente alérgicos que han desarrollado tolerancia alimentaria, no necesariamente produce recurrencia o agravación de la 
DA. Por cuanto los signos y síntomas de la exposición a alergenos alimentarios son muy variados y faltan estudios de evitación de los antígenos alimentarios relevantes bien diseñados en pacientes con DA, persiste la controversia si la AA puede exacerbar la DA. Una revisión sistemática que evaluó 9 estudios randomizados controlados ${ }^{39}$ para valorar los efectos de dieta de exclusión en el tratamiento de la DA confirmada en enfermos no seleccionados encontró escasa evidencia para confirmar el papel de la suspensión de alimentos. Sin embargo, otros estudios ${ }^{40,41}$ encontraron disminución del prurito cuando se suspendió el huevo en la dieta de pacientes con alergia al huevo y DA. En otro estudio sobre la historia natural de AA en niños menores de 18 meses con DA sometidos a dieta de eliminación estricta y pruebas de provocación oral durante un seguimiento de 1 a 2 años, el $26 \%$ no tuvo ninguna evidencia de $\mathrm{AA}^{21}$. En los niños que desarrollaron tolerancia al alimento específico se pudo reintroducir el alimento en la dieta sin recurrencia de síntomas ni empeoramiento de la DA en un seguimiento entre 6 meses y 4 años. Los niños que mostraron síntomas cutáneos y respiratorios durante la provocación oral al inicio del estudio, tuvieron mayor probabilidad de curarse de la AA en comparación con los niños que al inicio tuvieron sólo síntomas cutáneos o asociación de síntomas en piel y gastrointestinales.

Cuando la asociación DA-AA está bien confirmada deben tratarse en forma simultanea ambas condiciones. Cuidados de la piel, lubricación, emolientes, evitación de irritantes y fármacos tópicos o sistémicos en forma escalonada, en forma conjunta con dieta de eliminación estricta y prolongada (mínimo $8 \mathrm{sem}$ ) de los alergenos alimentarios identificados como relevantes, educación sobre contenido y rotulación de alimentos envasados, y en caso de alergia a proteína de leche de vaca fórmulas extensamente hidrolizadas o en base a aminoácidos según la gravedad, y corticoides u otros inmunosupresores en casos severos.

\section{Prevención}

A la fecha no hay estrategia terapéutica demostrada como útil para evitar el desarrollo de atopia en los individuos predispuestos, sin embargo, estudios proponen la lactancia materna exclusiva por 3 a 4 meses o el uso de formulas extensamente hidrolizadas en niños con alto riesgo de atopia.

Un estudio randomizado controlado mostró que los recién nacidos con historia familiar de atopia alimentados con fórmula tenían mayor riesgo de reacciones alérgicas especialmente $\mathrm{DA}$, en comparación con niños con lactancia materna ${ }^{42}$.

El uso de fórmula de soya durante los primeros 6 meses de edad en lactantes sin clínica de AA no demostró diferencias significativas en la incidencia de alergias, asma, rinitis o DA en comparación con leche materna, fórmulas hidrolizadas o leche de vaca según una revisión que incluyó tres estudios ${ }^{43}$.

Los beneficios de la intervención dietética en la prevención o retraso de la expresión de enfermedades atópicas se limitan a lactantes con riesgo de desarrollar alergia. La restricción de la dieta materna durante el embarazo o lactancia no tiene evidencias, en cambio la lactancia materna por lo menos durante 4 meses previene o retarda la DA, la alergia a leche de vaca y las sibilancias en los primeros meses de vida en comparación con fórmulas con leche de vaca entera. En lactantes con riesgo de enfermedades alérgicas, especialmente DA, alimentados con lactancia materna no exclusiva durante los primeros 4 a 6 meses, el empleo de fórmulas hidrolizadas tiene un efecto marginal en retardar o prevenir alergia cuando se compara con leche de vaca. No todas las fórmulas hidrolizadas muestran efectos protectivos, existe escasa evidencia que el retraso en la incorporación de alimentación complementaria más allá de los 4 a 6 meses previene el inicio de enfermedades atópicas y no hay datos concluyentes de un efecto protector para recomendar restricciones en la dieta después de los 4 a 6 meses.

Un metanálisis que comparó alimentación prolongada con fórmulas hidrolizadas versus alimentación con leche de vaca en lactantes con factores de riesgo encontró disminución significativa de alergia en lactantes en los niños alimentados con fórmulas hidrolizadas, pero no hubo diferencias en la incidencia de 
alergias (DA, asma, rinitis y AA) en niños mayores $^{44}$.

\section{Conclusiones}

La DA es una enfermedad inflamatoria de la piel, pruriginosa, crónica y recidivante con distribución universal, de curso y pronóstico variable, que ocasiona trastornos físicos y emocionales al paciente y su familia. La mayoría de los casos comienza en la edad pediátrica. En las tres últimas décadas ha tenido un incremento sustancial principalmente en países industrializados, por razones aún no bien esclarecidas.

Un subgrupo de pacientes con DA presentan AA, sin embargo, predomina el grupo que sólo presenta sensibilización no clínicamente relevante a ciertos antígenos alimentarios. $\mathrm{La}$ relevancia en el diagnóstico de DA y AA de los test cutáneos y serológicos es controversial debido a su poca estandarización y variación interpoblacional. Frente a AA el TPO se recomienda como herramienta diagnóstica y el mejor tratamiento actualmente corresponde a la dieta de eliminación.

La dieta de exclusión de los alimentos específicamente identificados está indicada en el tratamiento de enfermos con DA y AA confirmada por historia evidente o pruebas cutáneas y de provocación oral, ya que en enfermos con DA no seleccionados, la eliminación de alimentos en la dieta tiene escasos resultados favorables y en cambio puede ocasionar alteraciones nutricionales. La dieta de eliminación es capaz de disminuir la severidad de la DA, pero no se ha demostrado que modifique el curso de la enfermedad.

La dieta de exclusión tampoco ha mostrado beneficios como medida preventiva de desarrollar DA cuando se ha indicado a madres durante la lactancia.

El uso de fórmulas artificiales en niños con antecedentes familiares de atopia aumenta el riesgo de enfermedades alérgicas en comparación con niños que reciben lactancia materna. La evidencia no demuestra beneficio preventivo del uso de fórmulas en base a soya, y el uso de fórmulas con proteínas de leche de vaca hidrolizadas tiene escaso beneficio en prevenir o retrasar la sensibilización alérgica en niños con riesgo atópico. Como ya se comentó, no todas las fórmulas hidrolizadas muestran efectos protectivos similares, el retraso en la incorporación de alimentación complementaria más allá de $\operatorname{los} 4$ a 6 meses tiene escasa evidencia en la prevención de enfermedades atópicas y no hay datos concluyentes para recomendar restricciones en la dieta después de los 4 a 6 meses. El uso de fórmulas hidrolizadas versus alimentación con leche de vaca en lactantes con factores de riesgo encontró disminución significativa de alergia en lactantes, pero el beneficio no se extendió a edades mayores.

Actualmente no hay tratamiento para evitar la sensibilización cutánea en la DA. Están en marcha estudios que puedan demostrar la importancia de restaurar las alteraciones de la barrera cutánea y su rol en la prevención de sensibilización antigénica y en el desarrollo de enfermedades alérgicas, especialmente respiratorias ${ }^{45}$. Con estos antecedentes se recomienda tratar efectivamente las lesiones dérmicas para recuperar la función de barrera de la piel.

\section{Referencias}

1.- Greenhawt M: The role of food allergy in atopic dermatitis. Allergy Asthma Proc 2010; 31: 392-7.

2.- Worth A, Sheikh A: Food allergy and atopic eczema. Curr Opin Allergy Clin Immunol 2010; 10: 226-30.

3.- Allen K, Dharmage SC: The role of food allergy in the atopic march. Clin Exp Allergy 2010; 40: 1439-41.

4.- Adkis CA, Adkis M, Bieber T, et al: Diagnosis and treatment of atopic dermatitis in children and adults: European Academy of Allergology and Clinical Immunology/American Academy of Allergy, Asthma and Immunology/PRACTALL Consensus Report. J Allergy Clin Immunol 2006; 118: 152-69.

5.- Hahn EL, Bacharier LB: The Atopic March: The pattern of allergic disease development in childhood. Immunol Allergy Clin North Am 2005; 25: 231-46.

6.- Bieber T: Atopic dermatitis. Ann Dermatol 2010; 22: 125-37.

7.- Boyce JA, Assa'aa A, Burks AW, et al: Guidelines for the Diagnosis and Management of Food Allergy in the United States: Report of the NIAID-Sponsored Expert Panel. J Allergy Clin Immunol 2010; 126: S1-S58.

8.- Dokmeci E, Herrick CA: The immune system and atopic 
dermatitis. Semin Cutan Med Surg 2008; 27: 38-143.

9.- Brown SJ, Irvine AD: Atopic eczema and the filaggrin history. Semin Cutan Med Surg 2008; 27: 128-37.

10.- Kupper TS, Fuhlbrigge RC: Immune surveillance in the skin: mechanisms and clinical consequences. Nature Immunol 2004; 4: 211-22.

11.- Bieber T: Atopic dermatitis. N Engl J Med 2008; 358: 1483-94.

12.- Leung DYM, Jain N, Leo HL: New concepts in the pathogenesis of atopic dermatitis Curr Opin Immunol 2003; 15: 634-8.

13.- Hata TR, Gallo RL: Antimicrobial peptides, skin infections, and atopic dermatitis. Semin Cutan Med Surg 2008; 27: 144-50.

14.- Hill DJ, Hosking CS: Food allergy and atopic dermatitis in infancy: an epidemiologic study. Pediatr Allergy Immunol 2004; 15: 421-7.

15.- Novak N, Bieber T: The role of dendritic cells in atopic dermatitis. Clin Exp Allerg Rev 2004; 4: 140-5.

16.- Kim JS: Pediatric Atopic Dermatitis: The importance of food allergens. Semin Cutan Med Surg 2008; 27: 15660.

17.- Simon D, Braathen LR, Simon HU: Eosinophils and atopic dermatitis. Allergy 2004; 59: 561-70.

18.- Tokura Y: Extrinsic and intrinsic types of atopic dermatitis. J Dermatol Sci 2010; 58: 1-7.

19.- Skolnick HS, Conover-Walker MK, Koerner CB, Sampson HA, Burks W, Wood RA: The natural history of peanut allergy. J Allergy Clin Immunol 2001; 107: 367-74.

20.- Skripak JM, Matsui EC, Mudd K, Wood RA: The natural history of IgE-mediated cow's milk allergy. J Allergy Clin Immunol 2007; 120: 1172-7.

21.- Sampson HA, Scanlon SM: Natural history of food hypersensitivity in children with atopic dermatitis. J Pediatr 1989; 115: 23-7.

22.- Eigenmann PA, Sicherer SH, Borkowski TA, Cohen BA, Sampson HA: Prevalence of IgE-mediated food allergy among children with atopic dermatitis. Pediatrics 1998; 101: e8.

23.- van den Oord RA, Sheikh A: Filaggrin gene defects and risk of developing allergic sensitisation and allergic disorders: systematic review and meta-analysis. BMJ 2009; 339: b2433.

24.- Burks $W$ : Skin manifestations of food allergy. Pediatrics 2003; 111: 1617-24.

25.- Liacouras CA, Furuta GT, Hirano I, et al: Eosinophilic esophagitis: Updated consensus recommendations for children and adults. J Allergy Clin Immunol 2011; 128 : $3-20$.
26.- Mehr S, Kakakios A, Frith K, Kemp AS: Food proteininduced enterocolitis syndrome: 16-year experience. Pediatrics 2009; 123: e459-64.

27.- Leonard SA, Nowak-Wegrzyn A: Food protein-induced enterocolitis syndrome: an update on natural history and review of management. Ann Allergy Asthma Immunol 2011; 107: 95-101.

28.- Nowak-Wegrzyna A, Muraro A: Food protein-induced enterocolitis syndrome. Curr Opin Allergy Clin Immunol 2009; 9: 371-7.

29.- Hanifin JM, Chan SC, Cheng JB, et al: Type 4 phosphodiesterase inhibitors have clinical and in vitro effects in atopic dermatitis. J Invest Dermatol 1996; 107: 51-6.

30.- Sampson HA, Jolie PL: Increased plasma histamine concentrations after food challenges in children with atopic dermatitis. N Engl J Med 1984; 311: 372-4.

31.- Green TD, LaBelle VS, Steele PH, et al: Clinical characteristics of peanut-allergic children: recent changes. Pediatrics 2007; 120: 1304-10.

32.- Hill DJ, Hosking CS, de Benedictis FM, Oranje AP, Diepgen TL, Bauchau V: Confirmation of the association between high levels of immunoglobulin $\mathrm{E}$ food sensitization and eczema in infancy: an international study. Clin Exp Allergy 2008; 38: 161-8.

33.- Fox AT, Sasieni P, Du Toit G, Syed H, Lack G: Household peanut consumption as a risk factor for the development of peanut allergy. J Allergy Clin Immunol 2009; 123: 417-23.

34.- Vogel NM, Katz HT, Lopez R, Lang DM: Food allergy is associated with potentially fatal childhood asthma. $\mathrm{J}$ Asthma 2008; 45: 862-6.

35.- Bock SA, Muñoz-Furlong A, Sampson HA: Fatalities due to anaphylactic reactions to foods. J Allergy Clin Immunol 2001; 107: 191-3.

36.- Bock SA, Sampson HA: Immediate reactions to foods in infants and children. En: Metcalfe D, Sampson H, Simon R. edit. Food Allergy: Adverse Reactions to Foods and Food Additives, 4th edition, Blackwell Publishing, New York, 2008

37.- Bath-Hextall F, Delamere FM, Williams HC: Dietary exclusions for established atopic eczema. Cochrane Database Syst Rev 2008 art. no. CD005203.

38.- Kramer MS, Kakuma R: Maternal dietary antigen avoidance during pregnancy or lactation, or both, for preventing or treating atopic disease in the child. Cochrane Database Syst Rev 2006 art. no. CD000133.

39.- Bath-Hextall F, Delamere FM, Williams HC: Dietary exclusions for improving established atopic eczema in adults and children: systematic review. Allergy 2009; 64: 258-64. 
40.- Agata H, Kondo N, Fukutomi O, Shinoda S, Orii T: Effect of elimination diets on food-specific $\operatorname{IgE}$ antibodies and lymphocyte proliferative responses to food antigens in atopic dermatitis patients exhibiting sensitivity to food allergens. J Allergy Clin Immunol 1993; 91: 668-79.

41.- Lever R, MacDonald C, Waugh P, Aitchison T: Randomised controlled trial of advice on an egg exclusion diet in young children with atopic eczema and sensitivity to eggs. Pediatr Allergy Immunol 1998; 9: 13-9.

42.- Lucas A, Brooke OG, Morley R, Cole TJ, Bamford $M F$ : Early diet of preterm infants and development of allergic or atopic disease: randomised prospective study. BMJ 1990; 300: 837-40.

43.- Osborn DA, Sinn J: Soy formula for prevention of allergy and food intolerance in infants. Cochrane Database Syst Rev 2006 art. no. CD003741.

44.- Osborn DA, Sinn J: Formulas containing hydrolysed protein for prevention of allergy and food intolerance in infants. Cochrane Database Syst Rev 2006 art. no. CD003664.

45.- Lowe AJ, Abramson MJ, Hosking CS, et al: The temporal sequence of allergic sensitization and onset of infantile. Clin Exp Allergy 2007; 37: 536-42. 